\title{
Species-specific vesicular monoamine transporter 2 (VMAT2) expression in mammalian pancreatic beta cells: implications for optimising radioligand-based human beta cell mass (BCM) imaging in animal models
}

\author{
M. K.-H. Schäfer • N. R. Hartwig • N. Kalmbach • \\ M. Klietz • M. Anlauf • L. E. Eiden • E. Weihe
}

Received: 5 September 2012 / Accepted: 11 January 2013 /Published online: 13 February 2013

(C) Springer-Verlag Berlin Heidelberg 2013

\begin{abstract}
Aims/hypothesis Imaging of beta cell mass (BCM) is a major challenge in diabetes research. The vesicular monoamine transporter 2 (VMAT2) is abundantly expressed in human beta cells. Radiolabelled analogues of tetrabenazine (TBZ; a low-molecular-weight, cell-permeant VMAT2selective ligand) have been employed for pancreatic islet imaging in humans. Since reports on TBZ-based VMAT2 imaging in rodent pancreas have been fraught with confusion, we compared VMAT2 gene expression patterns in the mouse, rat, pig and human pancreas, to identify appropriate animal models with which to further validate and optimise TBZ imaging in humans.

Methods We used a panel of highly sensitive VMAT2 antibodies developed against equivalently antigenic regions of the transporter from each species in combination with
\end{abstract}

Electronic supplementary material The online version of this article (doi:10.1007/s00125-013-2847-7) contains peer-reviewed but unedited supplementary material, which is available to authorised users.

M. K.-H. Schäfer $(\bowtie) \cdot$ N. R. Hartwig $\cdot$ N. Kalmbach •

M. Klietz $\cdot$ E. Weihe

Department of Molecular Neuroscience, Institute of Anatomy and Cell Biology, Philipps University Marburg, Robert-Koch-Straße 8, 35037 Marburg, Germany

e-mail: mkh.schafer@staff.uni-marburg.de

M. Anlauf

Section on Neuroendocrine Neoplasms, Department of Pathology and Biomaterial Bank of the University Cancer Center,

University of Düsseldorf, Düsseldorf, Germany

L. E. Eiden

Section on Molecular Neuroscience, NIMH, Bethesda, MD, USA immunostaining for insulin and species-specific in situ hybridisation probes. Individual pancreatic islets were obtained by laser-capture microdissection and subjected to analysis of mRNA expression of VMAT2.

Results The VMAT2 protein was not expressed in beta cells in the adult pancreas of common mouse or rat laboratory strains, in contrast to its expression in beta cells (but not other pancreatic endocrine cell types) in the pancreas of pigs and humans. VMAT2- and tyrosine hydroxylase co-positive (catecholaminergic) innervation was less abundant in humans than in rodents. VMAT2-positive mast cells were identified in the pancreas of all species.

Conclusions/interpretation Primates and pigs are suitable models for TBZ imaging of beta cells. Rodents, because of a complete lack of VMAT2 expression in the endocrine pancreas, are a 'null' model for assessing interference with BCM measurements by VMAT2-positive mast cells and sympathetic innervation in the pancreas.

Keywords Beta cell mass · Imaging · Pancreas · Tetrabenazine $\cdot$ Vesicular monoamine transporter

$\begin{array}{ll}\text { Abbreviations } \\ \text { Acc.Nr. } & \text { GenBank accession number } \\ \text { BCM } & \text { Beta cell mass } \\ \text { DTBZ } & \text { Dihydrotetrabenazine } \\ \text { LCM } & \text { Laser-capture microdissection } \\ \text { nt. } & \text { Nucleotides } \\ \text { PET } & \text { Positron emission tomography } \\ \text { PP } & \text { Pancreatic polypeptide } \\ \text { SSC } & \text { Sodium citrate, sodium chloride buffer } \\ \text { TBZ } & \text { Tetrabenazine } \\ \text { VMAT } & \text { Vesicular monoamine transporter }\end{array}$




\section{Introduction}

We and others have previously identified the catecholamine transporters VMAT1 and VMAT2 (vesicular monoamine transporter 1 and 2) and characterised their expression patterns in rodents [1, 2] and in primates [3]. VMAT2 is the only vesicular monoamine transporter produced in the brain and in postganglionic sympathetic neurons in all species examined [4]. VMAT1 is likewise invariably expressed in the adrenal medulla and small intensely fluorescent cells in mammals; however, there is pronounced mammalian species variation in the expression of VMAT2 in endocrine tissues [3]. Thus, while VMAT1 is the major vesicular catecholamine transporter in the adrenal medulla of rodent species, VMAT1 and VMAT2 are co-expressed in chromaffin cells in primate species $[5,6]$ and VMAT2 is predominantly expressed in the bovine adrenal medulla [7,8]. VMAT1 and VMAT2 are both transporters for serotonin, dopamine, noradrenaline (norepinephrine) and adrenaline (epinephrine), and the function of both is blocked by reserpine, while VMAT2 also transports histamine and binds tetrabenazine (TBZ) [9]. The different properties of VMAT1 and VMAT2 are exhibited in enterochromaffin cells of the gut, which express VMAT1 and accumulate serotonin but not histamine, and enterochromaffin-like cells of the oxyntic mucosa of the stomach, which produce VMAT2 and accumulate histamine [5].

Although the beta cell is not known to synthesise, store and release biogenic amines, we previously demonstrated that VMAT2 is produced in most, if not all, human and nonhuman primate beta cells $[5,10]$, and this finding has been copiously confirmed $[11,12]$. Therefore, VMAT2 is a putative molecular target for the quantitative imaging of pancreatic beta cell mass (BCM) in diabetic patients [13-16]. Although VMAT2 is expressed within the cell, rather than on the cell surface, it can be labelled in vivo with the lipophilic positron emission tomography (PET) ligand $\left[{ }^{11} \mathrm{C}\right]$ dihydrotetrabenazine (DTBZ). Several groups have used $\left[{ }^{11} \mathrm{C}\right] \mathrm{DTBZ}$ to estimate the density of VMAT2-positive nerve terminals in the human brain in neurodegenerative disorders such as Parkinson's disease $[17,18]$, and the dynamics of VMAT2 occupancy by catecholamines in methamphetamine users has also been probed using labelled DTBZ [19]. More recently, $\left[{ }^{11} \mathrm{C}\right] \mathrm{DTBZ}$ has been employed for PET analysis of BCM in humans. However, animal models have been deemed critical for optimising the chemistry and pharmacodynamics of DTBZ analogues, to accelerate their widespread acceptance in clinical practice, as markers that might be used even more widely for imaging of BCM in human patients at critical milestones in the progression of a disease, such as its diagnosis, determination of its prognosis and assessment of therapy.

Rats, mice and pigs have all been used as models for DTBZ imaging of beta cells but the interpretation of the results of rodent DTBZ imaging in the pancreas has been controversial [20-29]. This is partly because the biochemical, histochemical and molecular biological characterisation of VMAT2 expression in the pancreas in these species, compared with humans, has yet to be rigorously examined. Therefore, we investigated here the extent to which VMAT2 is expressed in beta cells of rodents in comparison with humans to qualify rodents as suitable animal models for in vivo BCM imaging with TBZ-based PET tracers. In addition, we evaluated the abundance of VMAT2 in pancreatic cellular compartments other than beta cells, including pancreatic nerves and mast cells, as being a possible contributor to PET signals obtained with TBZ tracer. Finally, we examined VMAT2 expression in other species that are commonly used as animal models in preclinical studies.

\section{Methods}

Animals and tissue samples Pancreatic tissues from adult male mice $(\mathrm{C} 57 / \mathrm{bl6}, \mathrm{BALB} / \mathrm{c}, \mathrm{C} 3 \mathrm{H} / \mathrm{HeN})$ and rats (Wistar, Sprague-Dawley, Lewis) of various strains (Charles River, Sulzfeld, Germany) were rapidly collected in accordance with the German Law for animal protection. Immediately after the rodents were killed by exposure to isoflurane, tissue samples were collected and were frozen in liquid nitrogen (for RNA extraction) or isopentane (for preparing cryosections) or were immersed overnight in Bouin Hollande fixative or freshly prepared paraformaldehyde (for immunocytochemical processing). Human pancreatic tissue sections were prepared as described previously [10]. Four frozen normal human pancreas specimens, containing nonpathological endocrine islets, were provided by the Biomaterial Bank of the University Cancer Center of Düsseldorf, Germany. The tissue was taken from patients (aged 4771 years) suffering from pancreatic adenocarcinoma in approval with the local ethics committee. Porcine adrenal and pancreatic tissues were collected immediately post-mortem from adult pigs in the local slaughter house. In addition tissues from slaughtered 3-month-old female domestic pigs (Sus scrofa domestica; Lehr- und Forschungsstation Oberer Hardthof, Justus Liebig University, Giessen, Germany) were provided by T. Steinfeldt (Department of Anaesthesiology and Critical Care, University Hospital Giessen-Marburg, Philipps University Marburg) in accordance with the Principles of Laboratory Care. Tissues were cut into small blocks with a side length of less than $10 \mathrm{~mm}$ and processed as described above.

Laser-capture microdissection Using the P.A.L.M. Microbeam system (Carl Zeiss, Munich, Germany), laser-capture microdissection (LCM) was performed on frozen counterstained sections of mouse and pig pancreas and adrenal 
glands. LCM of human pancreas was carried out under fluorescent illumination (488 $\mathrm{nm}$ excitation wavelength) taking advantage of the autofluorescent properties of human beta cells [30]. The detailed procedures are described in electronic supplementary material (ESM) Methods.

$R T-P C R$ Total RNA from tissues was isolated using TRIzol Reagent (Invitrogen, Darmstadt, Germany). Isolation of total RNA from microdissected islets was performed with a micro-scale RNA isolation kit (RNAqueous-Micro; Ambion, Austin, Texas). All RNA samples were treated with RNase-free DNase I (Roche Diagnostics, Mannheim, Germany) at $37^{\circ} \mathrm{C}$ for $30 \mathrm{~min}$. RNA quality was measured by capillary gel electrophoresis (Experion Bioanalyzer; BioRad Medical Diagnostics GmbH, Dreieich, Germany). Details are described in ESM Methods. Synthesis of cDNA and PCR was performed as described previously [31]. All cDNA was tested for genomic DNA contamination by omitting reverse transcriptase in the synthesis reaction. Geneand species-specific primer pairs are listed in ESM Table 1. Identity of all PCR products were confirmed by doublestranded DNA sequencing (SEQlab, Göttingen, Germany). A standard PCR protocol was used with an annealing temperature of $60^{\circ} \mathrm{C}$. After $30-35$ cycles $10 \mu \mathrm{l}$ of each PCR reaction (LCM samples, 40 cycles) were loaded and electrophoresed on $1.5 \%$ agarose gels containing ethidium bromide and visualised by ultraviolet transillumination (BioRad, Munich, Germany).

Cloning of porcine VMAT2 VMAT2 (also known as SLC18A2) cDNA was cloned from the adrenal medulla of Sus scrofa domestica using a PCR cloning strategy. Two porcine expressed sequence tags (ESTs) were identified in GenBank with $87 \%$ homology to the $5^{\prime}$ end (GenBank accession No. [Acc.Nr.] CN158265.1) and 92\% homology to the 3' end (Acc.Nr. CN160191.1) of human VMAT2 (Acc.Nr. NM_003054.4) and used for primer design. After reverse transcription of high-quality porcine adrenal RNA (RNA quality index [RQI] >9) with Superscript III (Invitrogen), PCR was performed with PfuUltra High Fidelity DNA Polymerase (Agilent Technologies, Waldbronn, Germany) using the following primer pair: forward CAGGG CAGGCAGCCGCAGG; reverse TCACTTTCACCAG GGATGAGCGG. Sequence identities of amplicons from three independent PCR reactions were determined by custom double-stranded DNA sequencing (Seqlab, Göttingen, Germany). A cDNA (1,701 bp) containing the full-length coding sequence of porcine VMAT2 mRNA was obtained (Acc.Nr. KC552360), which was $100 \%$ identical to the VMAT2 mRNA sequence predicted by computational analysis (Acc.Nr. XM 001927394.3). The deduced sequence codes for the 517-amino-acid-long protein, which shares 93\% homology to human VMAT2, are shown in ESM Fig. 1.
Generation of DNA templates for in situ hybridisation probes Species-specific DNA templates for VMAT2 were generated as follows. A 734-bp-long mouse-specific Vmat2 cDNA (nucleotides [nt.]1076-1809; Acc.Nr. NM_17253) was obtained (Acc.Nr. NM 17253) by RT-PCR from brain cDNA extracts. A 368-bp-long BamHI/XbaI cDNA fragment of rat Vmat2 (nt. 233-500; Acc.Nr. L00603.1) was subcloned into pCRII [32]. A full-length human VMAT2 cDNA (Acc.Nr. L23205) was used as PCR template to generate two cDNA probes directed against the $5^{\prime}$ end (nt. 113-878) and the $3^{\prime}$ end (nt. 9,070-1,769), respectively. Similarly, the cloned pig VMAT2 cDNA served as template to produce a 727-bp-long 5'-specific and a 731-bp-long 3'specific fragment. For detection of mRNA for the genes encoding insulin and glucagon in the mouse, a 630-bplong cDNA (nt. 332-661, Acc.Nr. NM_00838.6) and a 545-bp-long cDNA (nt. 155-699, Acc.Nr. NM_008100.3), respectively, were generated by RT-PCR cloning from mouse pancreas. All DNA fragments were sublconed into pGEM-T (Promega, Mannheim, Germany), if not otherwise stated, for in vitro transcription to synthesise RNA probes in anti-sense- and sense-strand orientation using the appropriate RNA polymerases SP6 and T7 as described [33].

In situ hybridisation Frozen sections of pancreas $(10 \mu \mathrm{m}$ thick) from all species examined were cut on a LEICA cryostat, thaw-mounted on adhesive slides and subjected to the hybridisation procedure as described [33]. Sections were covered with $50 \mu \mathrm{l}$ hybridisation buffer containing $\left[{ }^{35} \mathrm{~S}\right] \mathrm{UTP}$ labelled riboprobes $\left(5 \times 10^{4} \mathrm{dpm} / \mu \mathrm{l}\right)$, coverslipped and incubated for $14 \mathrm{~h}$ at $60^{\circ} \mathrm{C}$ in a humid chamber. Slides were washed in decreasing concentrations of $2 \times$ SSC, RNase A-treated, dehydrated, air dried and then coated with NTB-2 nuclear emulsion (Eastman Kodak, Rochester, NY, USA). After exposure times between 4 and 28 days, slides were developed. Sections were analysed with the Olympus AX70 fluorescence microscope (Olympus Optical, Hamburg, Germany) and results were documented with a digital photographic camera system (Diagnostics Instruments, Ann Arbor, MI, USA).

Immunohistochemistry Animals were perfused with PBS and Bouin Hollande or freshly prepared $4 \%$ paraformaldehyde fixative. All antisera used had been characterised previously $[10,34]$ (ESM Table 2). Species-specific biotinylated secondary antibodies (1:200 working dilution; Dianova, Hamburg, Germany) were employed using the Vectastain ABC method (Vectastain Elite ABC Kit; Vector Laboratories, Burlingame, CA, USA), including ammonium nickel sulfate-enhanced 3,3diaminobenzidine (Sigma, Deisenhofen, Germany) reactions to enhance antibody visualisation.

Immunofluorescence and morphometric analysis After deparaffinisation and blocking procedures appropriate 
combinations of two primary antibodies raised in different donor species were co-applied in $\mathrm{PBS} / 1 \% \mathrm{BSA}$ and incubated overnight at $4^{\circ} \mathrm{C}$, followed by incubation for $2 \mathrm{~h}$ at $37^{\circ} \mathrm{C}$. After extensive washing in distilled water followed by PBS, immunoreactions for the first primary antibody were visualised with species-specific secondary antibodies labelled with Alexa Fluor 647 (MoBiTec, Göttingen, Germany), diluted 1:200 in 1\% BSA/PBS. The second primary antibody was visualised by a two-step procedure including species-specific biotinylated secondary antisera (Dianova), diluted 1:200 in 1\% BSA/PBS followed by streptavidin conjugated with Alexa Fluor 488, diluted $1: 100$ in $1 \% \mathrm{BSA} / \mathrm{PBS}$. Incubation times were $45 \mathrm{~min}$ with biotinylated secondary antibodies, followed by a $2 \mathrm{~h}$ incubation with a mixture of fluorochrome-conjugated secondary antibody and streptavidin. Biotinylated secondary antiserum incubations with the species-specific VMAT2 antibodies were followed by tyramide signal amplification using the individual indirect tyramide reagent pack (catalogue No. SAT700; PerkinElmer, Rodgau, Germany) and visualisation with streptavidin conjugated with Alexa Fluor 488 (dilution 1:200; MoBiTec, Göttingen, Germany). A directly labelled secondary antibody with Alexa Fluor 647 was applied simultaneously to the other primary antibody species for $2 \mathrm{~h}$ at $37^{\circ} \mathrm{C}$.

Immunofluorescent signals were documented as digitised false colour images (8-bit tiff format) with an Olympus BX50WI confocal laser scanning microscope (Olympus Optical, Hamburg, Germany) and Olympus Fluoview 2.1 software.

For morphometric analysis, serial sections $(n=9)$ from four different levels of human pancreas containing islets were stained with VMAT2 and tryptase. Pictures were taken with a $\times 10$ objective and analysed using Image $J$ software (National Institutes of Health, Bethesda, MD, USA).

\section{Results}

Vmat2 in situ hybridisation of rodent pancreas After hybridisation of pancreas sections with $\left[{ }^{35} \mathrm{~S}\right]$-labelled species-specific riboprobes for Vmat2 a complete absence of positive signals was observed over pancreatic islets and exocrine glands of mouse (Fig. 1a, b) and rat (Fig. 1d, e). This was in contrast to the very strong positive signals for proinsulin and proglucagon mRNA in sections from the same tissue under the same hybridisation conditions (ESM Fig. 2). As a positive Vmat2 probe control, the adrenal medulla of mice and rats obtained from the same animals from which the pancreas sections had been prepared was used (Fig. 1c, f). Strong signals for Vmat 2 mRNA were detected in subpopulations of adrenomedullary cells fully validating the Vmat2 in situ hybridisation probes to test
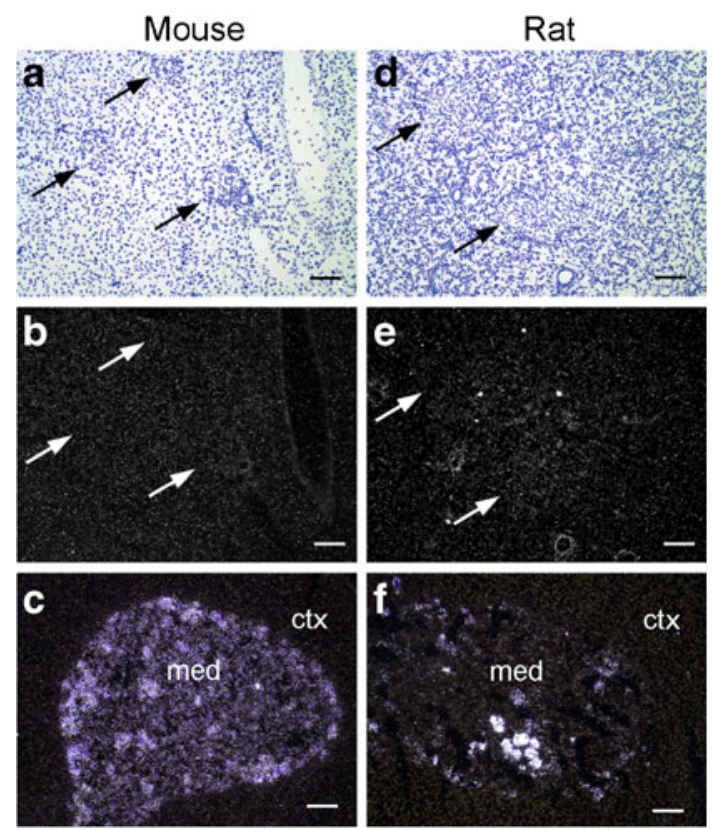

Fig. 1 Absence of Vmat2 mRNA expression in mouse and rat endocrine pancreas. Radioactive in situ hybridisation of mouse (a-c) and rat (d-f) pancreas and adrenal gland using species-specific ${ }^{35} \mathrm{~S}$-labelled riboprobes for Vmat $2 \mathrm{mRNA}$. Dark field autoradiograms of mouse (b) and rat (e) pancreas illustrate the absence of specific VMAT2 hybridisation signals in islets (arrows), which are identified under bright-field illumination by cresyl violet staining (arrows in a and $\mathbf{d}$ correspond to arrows in $\mathbf{b}$ and $\mathbf{e}$, respectively). VMAT2 signals are also undetectable in the exocrine pancreas $(\mathbf{c}, \mathbf{f})$. In contrast, VMAT2 probes produce strongly positive hybridisation signals in specific subpopulations of chromaffin cells in both mouse (c) and rat (f) adrenal medulla (med). Autoradiograms were exposed for 28 days. ctx, adrenal cortex; scale bars, $100 \mu \mathrm{m}$

pancreatic islet cells for rodent Vmat2 mRNA expression. The same results as those obtained for adult $\mathrm{C} 57 \mathrm{~B} 1 / 6$ mice and Wistar rats, shown in Fig. 1, were obtained using Balb/c, BVN2 and $\mathrm{C} 3 \mathrm{H} / \mathrm{HeN}$ mice and Sprague-Dawley and Lewis rats (data not shown).

Absence of Vmat2 mRNA in laser-microdissected mouse pancreatic islets The inability to detect Vmat2 mRNA signals by in situ hybridisation histochemistry in rodent pancreas indicated that the levels of Vmat2 mRNA are far below those previously reported for VMAT2 mRNA in human pancreatic islets. However, this does not rule out the possibility that the extremely low levels of Vmat 2 mRNA could potentially generate VMAT2 protein in endocrine pancreas, albeit at correspondingly low levels. To estimate whether very low levels of, or essentially no, VMAT2 is produced in the mouse endocrine pancreas, $\mathrm{C} 57 \mathrm{Bl} / 6$ mouse pancreatic islets of Langerhans were captured from $10 \mu \mathrm{m}$ frozen sections by LCM and analysed by RT-PCR (Fig. 2), after assurance of RNA integrity as described in the Methods. Total RNA was isolated and reverse transcribed using commercial kits (Invitrogen). PCR with two different primer sets 
for VMAT2 did not produce any amplicons in pancreatic islet cDNA samples, even after 40 cycles of amplification of cDNA input from approximately $1 \mu \mathrm{g}$ of isolated total RNA (Fig. 2b). Controls for efficiency of RNA isolation, cDNA synthesis and RT-PCR were carried out in two ways. First, RNA isolated by LCM from the pancreas, which is negative for Vmat2 mRNA, produced robust RT-PCR signals for proinsulin and proglucagon mRNA. Second, RNA isolated by LCM from the adrenal medulla, which is negative for proglucagon and proinsulin mRNA, produced robust RTPCR signals for Vmat2 mRNA (Fig. 2d).

Confocal analysis of VMAT2 immunoreactive structures in rodent pancreas VMAT2-positive nerve fibres were abundant in rodent pancreas and distributed throughout the endocrine and exocrine parts (Fig. 3a). VMAT2 immunoreactivity was completely absent from rodent endocrine cells (Fig. 3b). However, both mouse and rat islets received sympathetic VMAT2-coded innervation (Fig. 3b), indicating that the rat and mouse VMAT2 antibodies employed (see ESM Table 2) were appropriate to test for VMAT2 presence in islet endocrine cells, and that absence of VMAT2 from islet cells did not represent a false-negative result. VMAT2-positive mast cells were detected in rat and mouse pancreas, but made a relatively small contribution to overall VMAT2 protein levels in this organ. This is shown for mouse in Fig. 3c.

VMAT2 $m R N A$ expression in pig and human pancreas The actual molecular identity of recently reported TBZ ligand binding sites in pig pancreas [24] has not been established to be VMAT2. Accordingly, we identified the porcine adrenal
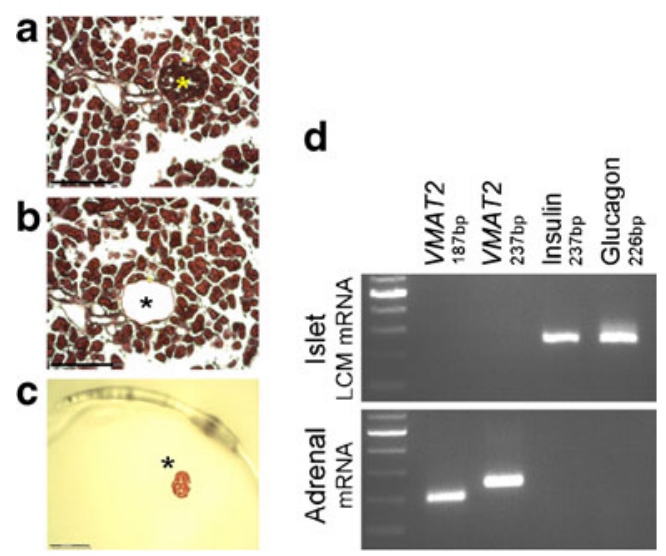

Fig. 2 PCR analysis of Vmat $2 \mathrm{mRNA}$ in laser-microdissected islets from mouse pancreas. $(\mathbf{a}-\mathbf{c})$ Endocrine islets were collected by LCM, as illustrated for one islet (asterisk in c). (d) PCR analysis with two different primer sets for Vmat2 revealed absence of Vmat2 transcripts in RNA extracts from LCM-isolated islets (40 PCR cycles). Note that in the same extract both insulin and glucagon transcripts are detectable already after 35 PCR cycles. Using microdissected mouse adrenal medulla as positive control RT-PCR yields specific Vmat2 amplicons with both Vmat 2 primer sets ( 35 cycles). See ESM Methods for further details; scale bars, $150 \mu \mathrm{m}(\mathbf{a}-\mathbf{c})$ a

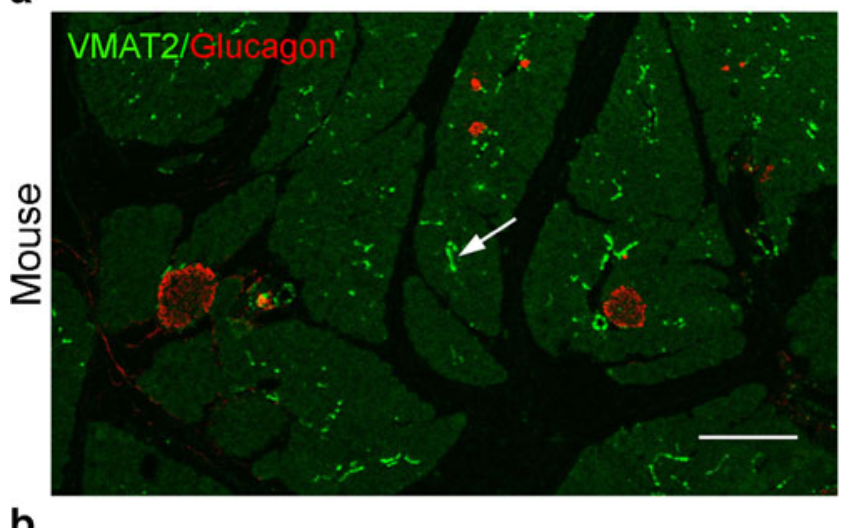

b

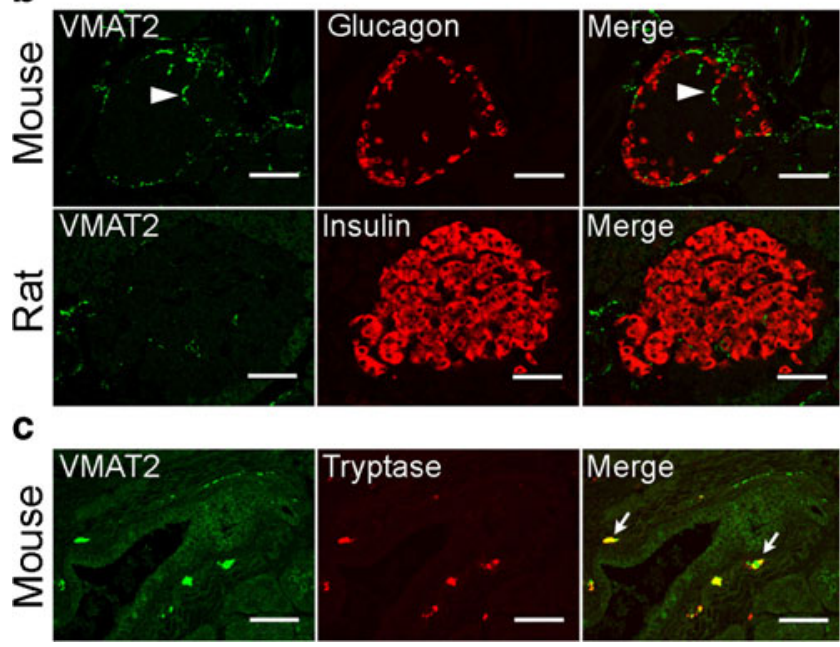

Fig. 3 VMAT2 protein visualisation in mouse pancreas: restriction to mast cells and nerve terminals. (a) Confocal double immunofluorescence microscopy for VMAT2 (green) reveals abundant VMAT2-positive varicose nerve fibres (arrow in a) scattered throughout the exocrine pancreas and innervating endocrine islets shown at low magnification for mouse pancreas and (b) high magnification for mouse and rat pancreas (merged images). Note the total absence of VMAT2 immunoreactivity in the endocrine cells of the pancreatic islets labelled red for glucagon and insulin, respectively; however, nerve fibres innervating the islets (arrowhead in b) are positive for VMAT2. (c) VMAT2 is present in periductal tryptase-positive mast cells (arrows) as well as in periductal nerves. Scale bars, $200 \mu \mathrm{m}(\mathbf{a}), 50 \mu \mathrm{m}(\mathbf{b}, \mathbf{c})$

medulla as a rich source of VMAT2 protein and VMAT2 mRNA using human-homologous reagents (data not shown). We then established the molecular identity of both messenger and protein via cloning of porcine VMAT2. Messenger RNA from porcine adrenal medulla amplified using highly species-conserved primers allowed us to obtain a sequence identical to the predicted open reading frame for VMAT2 within the established genomic sequence of porcine chromosome 14 (ESM Fig. 1). We confirmed that two nonoverlapping riboprobes derived from this sequence hybridised strongly and specifically to chromaffin cells of the porcine adrenal medulla (ESM Fig. 3). Species-specific riboprobes of similar length and composition were then used 
to compare porcine and human VMAT2 mRNA expression in the pancreas (Fig. 4). Both species exhibited islet-specific expression of VMAT2 mRNA, with the extent of mRNA expression in porcine islets (Fig. 4a) being somewhat less than in human islets (Fig. 4b).

VMAT2 mRNA in microdissected islet cells from pig and human pancreas LCM was employed to capture islet tissue from porcine pancreas, in parallel with human pancreas (Fig. 5). VMAT2 mRNA was obtained from approximately ten porcine or human islets from successive $10 \mu \mathrm{m}$ thick fresh frozen sections, in which islets were visualised either by counter-staining with cresyl violet (porcine) or by endogenous autofluorescence specific to islets compared with exocrine tissue (human). VMAT2 mRNA was easily detected, along with proglucagon and proinsulin mRNA, in both pig and human pancreatic islet RNA (Fig. 5d, h), using the same procedures that allowed detection of mRNA for proglucagon and proinsulin, but not Vmat $2 \mathrm{mRNA}$ in rodent pancreases (Fig. 2), and detection of VMAT2, but not proglucagon or proinsulin mRNA in porcine adrenal medulla (data not shown).

\section{Co-localisation of VMAT2 in beta cells of pig and human} pancreas VMAT2 was found in all islets of human pancreas, as shown here by comparative staining with antibodies against human VMAT2 and human chromogranin A on adjacent sections (Fig. 6a, b) and by double immunofluorescence for VMAT2 and insulin (Fig. 6c-h). To determine the appropriate antibodies for cell-specific co-production of
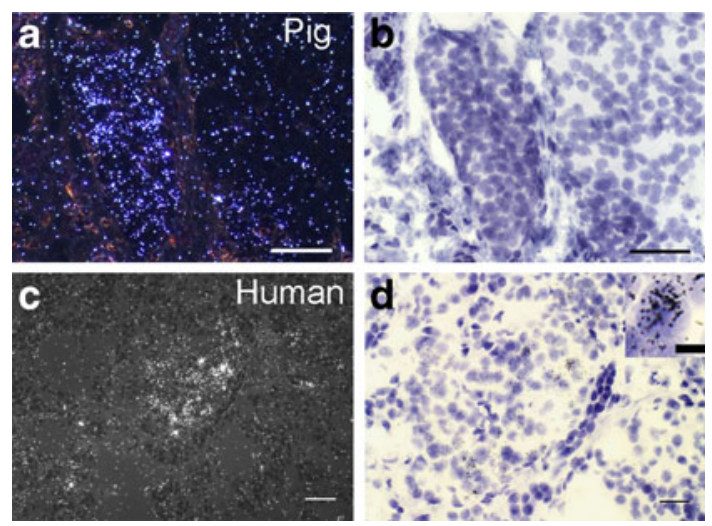

Fig. 4 VMAT2 in situ hybridisation histochemistry in pig and human pancreatic islets. Detection of VMAT2 mRNA in pig $(\mathbf{a}, \mathbf{b})$ and human (c, d) pancreas with species-specific ${ }^{35} \mathrm{~S}$-labelled riboprobes under dark field $(\mathbf{a}, \mathbf{c})$ and bright-field illumination $(\mathbf{b}, \mathbf{d})$. Positive hybridisation signals for VMAT2 (white silver grains in a, c; black grains in insert in d) are exclusively located in the central islet areas. Exocrine pancreatic regions of both pig and human display only randomly scattered signals representing background labelling. Exposure time, 21 days; scale bars, $100 \mu \mathrm{m}(\mathbf{a}, \mathbf{b}), 50 \mu \mathrm{m}(\mathbf{c}), 25 \mu \mathrm{m}(\mathbf{d}), 5 \mu \mathrm{m}$ (insert in d)
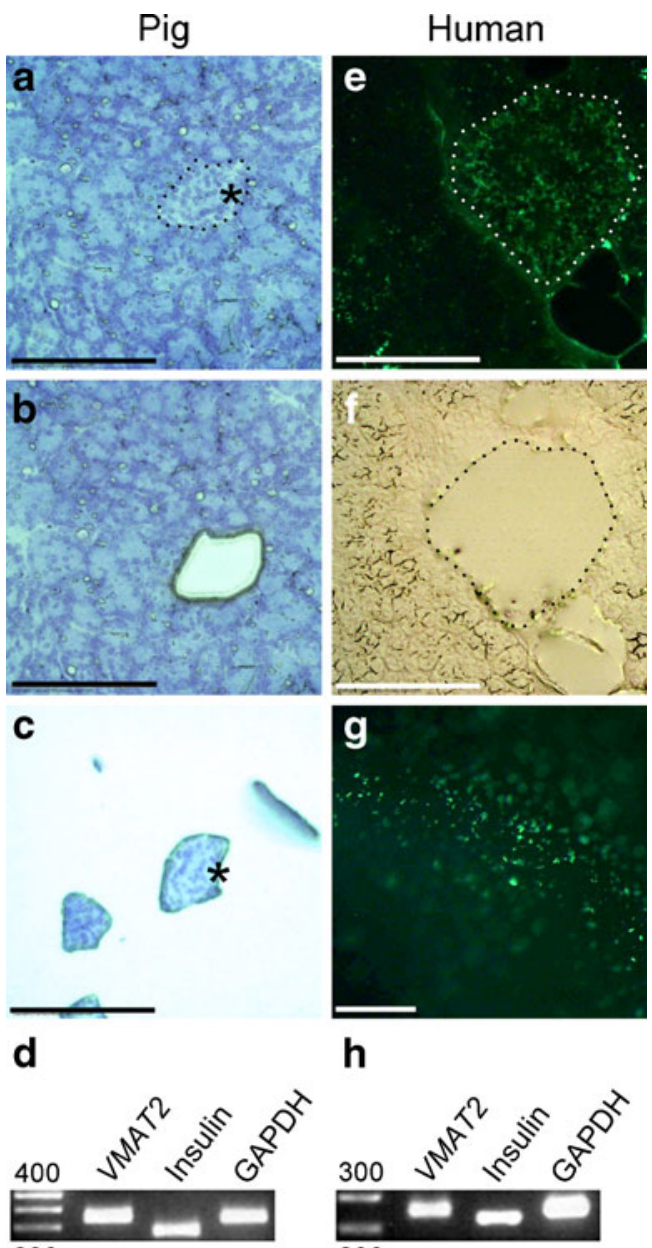

h

200

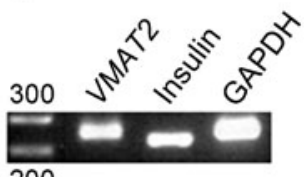

200

Fig. 5 Detection of VMAT2 mRNA in laser-microdissected islets of pig and human pancreas. (a-c) Pig islets were microdissected from cresyl violet-stained slides (asterisks). (e-g) Autofluorescent human islets were identified and microdissected under fluorescent illumination as illustrated for one islet. For further details see ESM Methods. (d, h) PCR analysis of pig (d) and human (h) islet extracts with speciesspecific primers reveals VMAT2 and insulin mRNA expression in the endocrine pancreas of both species. A universal GAPDH primer set was used as control PCR ( 35 cycles). Loaded volumes of $20 \mu \mathrm{l}$ PCR reactions were: (d) VMAT2 $15 \mu \mathrm{l}$, insulin $3 \mu \mathrm{l}$, GAPDH $10 \mu \mathrm{l}$; (h) VMAT2 $10 \mu \mathrm{l}, 3 \mu \mathrm{l}$, GAPDH $5 \mu \mathrm{l}$. Scale bars, $150 \mu \mathrm{m}$

VMAT2 within pancreatic islets of the pig, we compared the C-terminal sequence of porcine VMAT2 protein, within a highly antigenic region against which human-, rat- and mouse-specific antibodies had been previously generated. As depicted in ESM Table 2, porcine VMAT2 is identical, except for a single conserved amino acid (D for E), to human VMAT2 within the C-terminus of the protein. Therefore the previously generated anti-human VMAT2 antibody (80182) was employed for confocal immunofluorescence microscopy, with human pancreas as a positive control, to determine whether glucagon, somatostatin or pancreatic polypeptide (PP) cells might potentially contain low levels of VMAT2. We found that there was complete overlap 


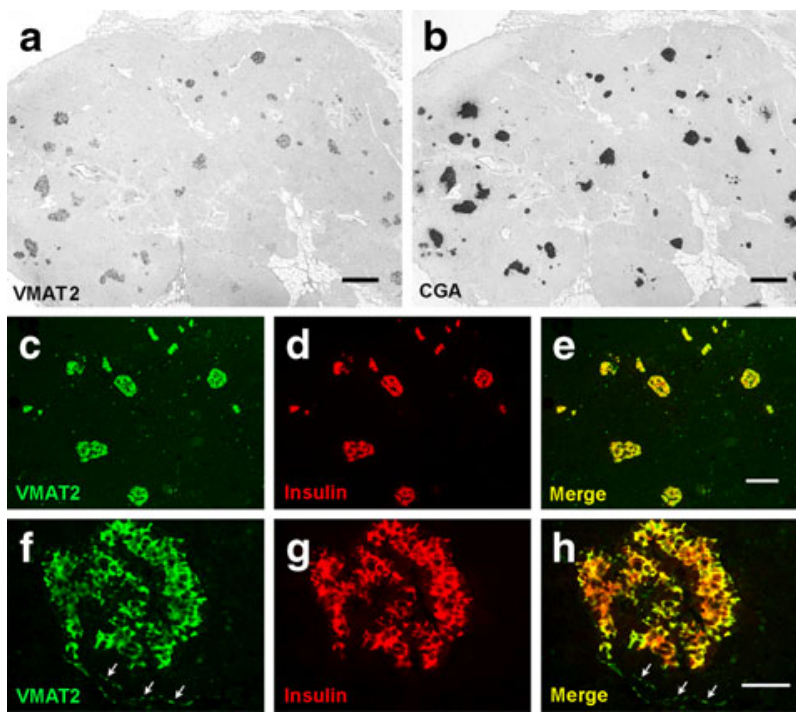

Fig. 6 Abundance of VMAT2 in human pancreatic islets. (a, b) Lowpower bright-field micrographs of a human pancreas from a nondiabetic man illustrate in adjacent sections that all large and small islet profiles staining for VMAT2 (a) also stain for the neuroendocrine pancreatic islet marker chromogranin A (CGA) (b). (c-e) Low-power confocal double immuofluorescence reveals full overlap of VMAT2stained small, large and tangential islet profiles with insulin-stained islet profiles. (f-h) Confocal double immunofluorescence at higher power reveals VMAT2-stained varicose nerve fibres (arrows in $\mathbf{f}, \mathbf{h}$ ) targeting the outer border of an islet with VMAT2/insulin co-positive beta cells. Scale bars, $500 \mu \mathrm{m}(\mathbf{a}, \mathbf{b}), 200 \mu \mathrm{m}(\mathbf{c}-\mathbf{e}), 50 \mu \mathrm{m}(\mathbf{f}-\mathbf{h})$

between VMAT2 and insulin presence in both pig and human endocrine pancreas (Fig. 7a) but we were unable to detect VMAT2 in glucagon- or somatostatin-positive cells in either species (Fig. 7b, c). As a rule, VMAT2 was absent from PP cells (Fig. 7d); only very rarely was co-staining for $\mathrm{PP}$ and VMAT2 seen.

Identification of VMAT2 in mast cells and in sympathetic innervation in human pancreas Throughout the exocrine pancreas, including periductal areas, VMAT2 was localised in mast cells as demonstrated by co-staining for the mast cell marker tryptase (Fig. 8a-f). The proportion of VMAT2 mast cell staining in pancreas amounted to about $10 \%$ of total VMAT2 staining in representative areas (Fig. 8g). Occasionally, single mast cells that were co-positive for VMAT2 and tryptase were encountered within the islet. VMAT2 was present in nerve fibres throughout the exocrine pancreas and in the vicinity of islets (Figs $6 \mathrm{f}, \mathrm{h}$, and $8 \mathrm{~d}$ ). Double immunofluorescence for VMAT2 and tyrosine hydroxylase identified the sympathetic nature of VMAT2 innervation throughout the pancreas as demonstrated for nerve profiles in juxtaposition to an islet (Fig. 9). Unlike in rodents, VMAT2/tyrosine hydroxylase co-positive innervation of islets was mostly restricted to the outer border of islets. Intra-islet fibres were confined to arterial blood vessel profiles. a

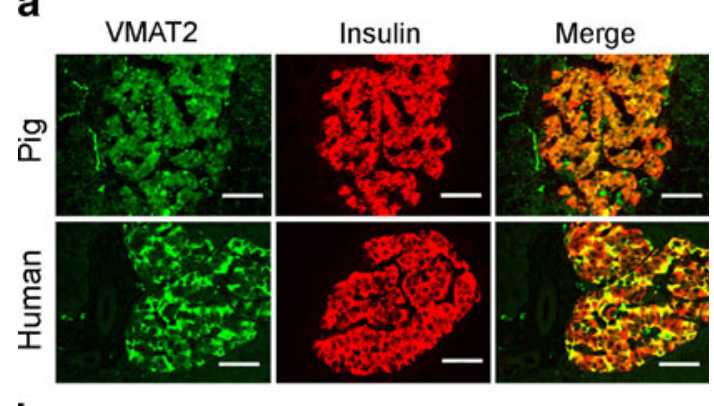

b

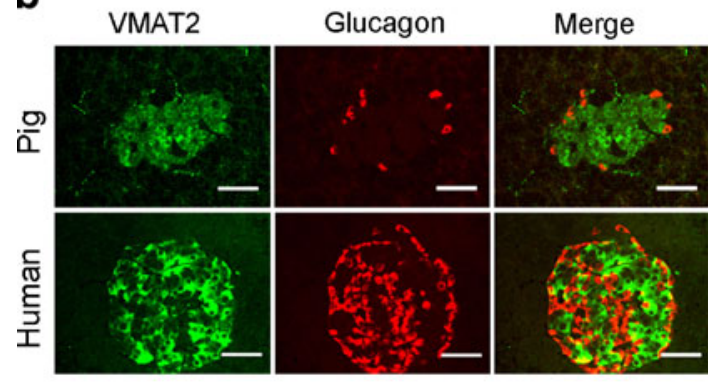

C

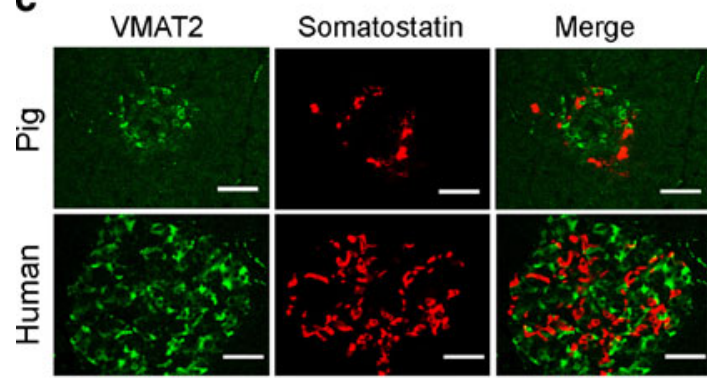

d

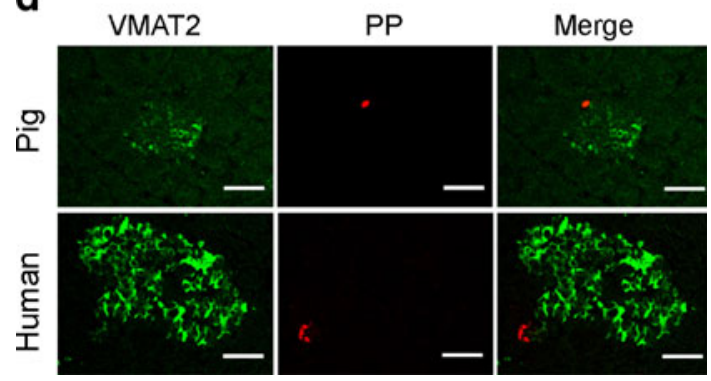

Fig. 7 VMAT2 co-localisation with insulin, but not glucagon, somatostatin or PP, in pig and human pancreas revealed by confocal double immunofluorescence. (a) In both pig and human islets VMAT2 and insulin immunostaining is always co-localised. Note that in both species there is mutual exclusion of VMAT2 staining and staining for glucagon (b), somatostatin (c) or PP (d). Scale bars, $50 \mu \mathrm{m}$

\section{Discussion}

The abundance of VMAT2 in pancreatic beta cells is remarkably species dependent. On the basis of the results reported here, we conclude that VMAT2 mRNA and VMAT2 protein is absent from beta cells of adult male rodent pancreatic islets but is present in pig and human beta cells. The relatively high abundance of pancreatic VMAT2 


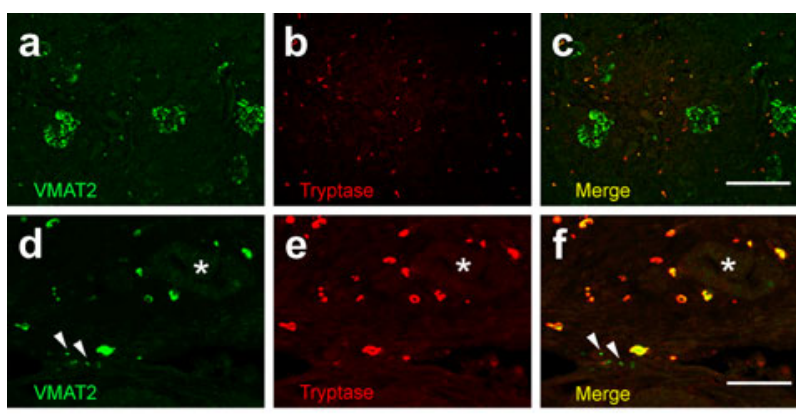

g

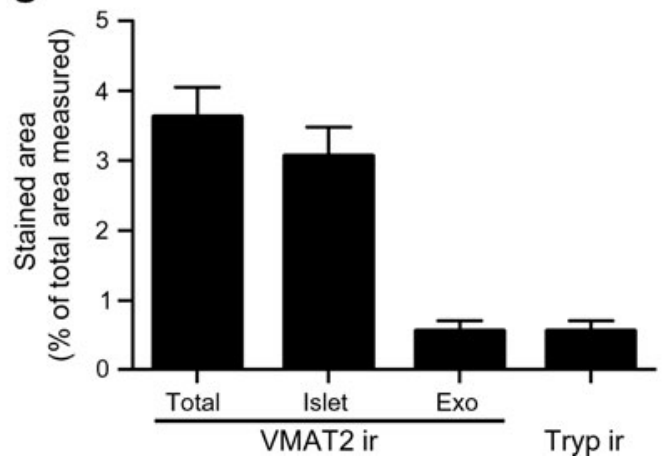

Fig. 8 Comparison of VMAT2 abundance in islets and non-islet structures (mast cells) of human pancreas. (a-c) Confocal double immunofluorescence at low power reveals VMAT2 in mast cells costained for the mast cell marker tryptase. Note VMAT2/tryptase copositive mast cells are located in juxtaposition to VMAT2-positive islet cells and also scattered throughout the exocrine pancreas. $(\mathbf{d}-\mathbf{f})$ Higherpower micrographs illustrate VMAT2/tryptase co-positive mast cells surrounding a pancreatic duct (indicated by asterisk) and VMAT2positive/tryptase negative nerve fibres (arrowheads) (g) Semiquantitative analysis of VMAT2 staining in human pancreas (stained area as a percentage of total view area): total VMAT2 immunoreactive (ir) area, $3.7 \pm 0.6 \%$; VMAT2 ir islet area, $3.3 \pm 0.7 \%$; VMAT ir exocrine (Exo) area, $0.4 \pm 0.1 \%$; tryptase (Tryp) ir area, $0.4 \pm 0.1 \%$. Scale bars, $200 \mu \mathrm{m}$ $(\mathbf{a}-\mathbf{c}), 50 \mu \mathrm{m}(\mathbf{d}-\mathbf{f})$

innervation in rodents (Fig. 3) as compared with humans (Figs 6,8) suggests that any specific pancreatic binding obtained with DTBZ-based PET tracers in rat or mouse is most likely related to VMAT2 in sympathetic nerves and mast cells present in both species. This may account for the lack of decrease in the small signal obtained even after treatment with streptozotocin, which results in beta cell loss in the rat pancreas [20]. Indeed, the presence of VMAT2 in
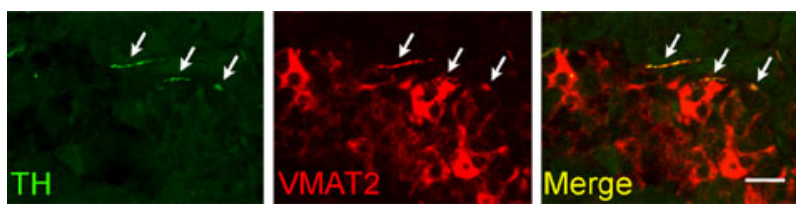

Fig. 9 Co-existence of tyrosine hydroxylase (TH) and VMAT2 in the sympathetic innervation of human pancreas. Confocal double immunofluorescence reveals longitudinal and cross profiles of nerve fibres (arrows) co-staining for TH (green) and VMAT2 (red) at the border of a pancreatic islet with VMAT2-positive beta cells. Scale bar, $20 \mu \mathrm{m}$ sympathetic nerves and mast cells throughout the pancreas, and species differences with regard to sympathetic innervation of the endocrine pancreas, must be taken into account in attempts to quantify BCM with DTBZ-based PET tracers in any species.

Rodents may be useful as animal models for pancreatic imaging with DTBZ PET tracers but only insofar as they provide controls for background and interfering signals other than VMAT2 presence in the pancreatic islets themselves. Importantly, the failure to adequately estimate BCM using DTBZ imaging in rodents does not reflect the lack of suitability of this ligand for imaging BCM in human patients, as some have suggested [20], but rather simply that the rodent is not the correct model for addressing this issue.

In humans we have shown that the relationship between the proportional areas of VMAT2-immunopositive beta cells and mast cells is about 9:1 implying that mast cells may confound estimation of BCM with DTBZ ligands only to a minor extent, if at all. However, as the PET signal reflects VMAT2 binding density, rather than area, the precise quantitative contribution of VMAT2 protein per mast cell and beta cell to which DTBZ ligands can bind in vivo remains to be shown. This information is obtainable only by imaging, modelled in transgenic rodents, or determined by quantitative topological analysis of in vivo DTBZ imaging itself.

So far, it appears that VMAT2 abundance in endocrine tissues is uniformly high or low depending on the species. Thus rodents produce little VMAT2 in the adrenal medulla and no VMAT2 in the endocrine pancreas, while primate VMAT2 abundance is relatively high in both the adrenal medulla and the endocrine pancreas. Consistent with these observations, we found VMAT2, by immunohistochemistry and by RT-PCR following LCM, to be present in both the adrenal medulla and the pancreatic islets of the pig.

Detailed examination reveals that, as in humans, VMAT2 presence in pig islets is confined to insulin-expressing beta cells. Importantly, neither glucagon-containing alpha cells nor somatostatin-containing delta cells in the endocrine pancreas of humans or pigs contain VMAT2, confirming the findings of our previous investigations in humans and non-human primates [10]. In contrast to a previous report [11], we only rarely observed VMAT2 expression in PP cells derived from human or pig pancreas. Given the small fraction of PP compared with insulin cells in the endocrine pancreas, and the relatively high and uniform VMAT2 level in the latter, it appears that this does not represent a major confounder in the estimation of human BCM using VMAT2 ligands.

In summary our findings indicate that the pancreatic islets of mice and rats do not contain VMAT2, except in the sympathetic nerve terminals that innervate the islets. An additional interesting possibility for examination of DTBZ binding using rodent models, however, is the development of transgenic expression of human VMAT2 in the mouse 
pancreas as a convenient imaging and biological model for studying the effect of VMAT2 level on beta cell function in humans. Porcine pancreas, like that of humans, contains VMAT2 in beta cells. Therefore the pig is a potential model, without transgenic manipulation, for estimation of BCM using TBZ analogues and has the further advantage of being frequently employed as an animal model in the study of cardiovascular and endocrine function in heart and metabolic disease states. Non-human primates are suitable models for DTBZ imaging of beta cells and, indeed, have been used by several laboratories for PET imaging of vesicular and plasma membrane biogenic amine transporters [35, 36]. Rodents, due to an absence of VMAT2 in islet cells, represent an interesting and possibly critical 'null' model for establishing a background for DTBZ imaging in the pancreas and for assessment of interference with BCM measurements by VMAT2-positive nerve terminals and mast cells. Development of Vmat2-transgenic rodent models for further analysis of DTBZ imaging of BCM could also be considered in a translational context. Finally, mammalian species specificity of VMAT2 production in beta cells is a timely and potent reminder that translational research in the development of biomarkers for human disease depends on the full and rigorous determination of the precise chemical anatomy of imaging targets not only in humans but in the species in which translation research leading to clinical development is carried forward.

Acknowledgements We are grateful to B. Wiegand, M. Schneider, H. Hlawaty and M. Zibuschka (Department of Molecular Neuroscience, Institute of Anatomy and Cell Biology, University of Marburg) for providing expert technical support and also to T. Steinfeldt (Department of Anaesthesiology and Critical Care, University Hospital GiessenMarburg, Philipps University Marburg) for collecting porcine tissue.

Funding Support by the BetaImage Consortium and EU grant EU FP7/-2013 No. 222980, and the University of Marburg Medical Faculty is gratefully acknowledged. L. E. Eiden acknowledges the NIMH Intramural Research program (under Project 1Z01MH002386) for research support.

Duality of interest The authors declare that there is no duality of interest associated with this manuscript.

Contribution statement MKHS, LEE and EW were responsible for the conception of the study, analysed and interpreted the data and wrote the manuscript. NRH, NK, MK and MA made substantial contributions to acquisition and interpretation of data and revised the manuscript. All authors approved the final version of the manuscript.

\section{References}

1. Weihe E, Schafer MK, Erickson JD, Eiden LE (1994) Localization of vesicular monoamine transporter isoforms (VMAT1 and
VMAT2) to endocrine cells and neurons in rat. J Mol Neurosci 5:149-164

2. Peter D, Liu Y, Sternini C, de Giorgio R, Brecha N, Edwards RH (1995) Differential expression of two vesicular monoamine transporters. J Neurosci 15:6179-6188

3. Erickson JD, Schäfer MK-H, Bonner TI, Eiden LE, Weihe E (1996) Distinct pharmacological properties and distribution in neurons and endocrine cells of two isoforms of the human vesicular monoamine transporter. Proc Natl Acad Sci USA 93:51665171

4. Eiden LE, Weihe E (2011) VMAT2: a dynamic regulator of brain monoaminergic neuronal function interacting with drugs of abuse. Ann N Y Acad Sci 1216:86-98

5. Eiden LE (2000) The vesicular neurotransmitter transporters: current perspectives and future prospects. FASEB J 14:2396-2400

6. Weihe E, Eiden LE (2000) Chemical neuroanatomy of the vesicular amine transporters. FASEB J 14:2435-2449

7. Howell M, Shirvan A, Stern-Bach Y et al (1994) Cloning and functional expression of a tetrabenazine sensitive vesicular monoamine transporter from bovine chromaffin granules. FEBS Lett 338:16-22

8. Krejjci E, Gasnier B, Botton D et al (1993) Expression and regulation of the bovine vesicular monoamine transporter gene. FEBS Lett 335:27-32

9. Eiden LE, Schafer MK, Weihe E, Schutz B (2004) The vesicular amine transporter family (SLC18): amine/proton antiporters required for vesicular accumulation and regulated exocytotic secretion of monoamines and acetylcholine. Pflugers Arch 447:636640

10. Anlauf M, Eissele R, Schafer MK et al (2003) Expression of the two isoforms of the vesicular monoamine transporter (VMAT1 and VMAT2) in the endocrine pancreas and pancreatic endocrine tumors. J Histochem Cytochem 51:1027-1040

11. Saisho Y, Harris PE, Butler AE et al (2008) Relationship between pancreatic vesicular monoamine transporter 2 (VMAT2) and insulin expression in human pancreas. J Mol Histol 39:543-551

12. Maffei A, Liu Z, Witkowski P et al (2004) Identification of tissuerestricted transcripts in human islets. Endocrinology 145:45134521

13. Kung HF, Lieberman BP, Zhuang ZP et al (2008) In vivo imaging of vesicular monoamine transporter 2 in pancreas using an (18)F epoxide derivative of tetrabenazine. Nucl Med Biol 35:825-837

14. Freeby M, Goland R, Ichise M, Maffei A, Leibel R, Harris P (2008) VMAT2 quantitation by PET as a biomarker for beta-cell mass in health and disease. Diabetes Obes Metab 10(suppl 4):98108

15. Simpson NR, Souza F, Witkowski P et al (2006) Visualizing pancreatic beta-cell mass with [11C]DTBZ. Nucl Med Biol 33:855-864

16. Normandin MD, Petersen KF, Ding YS et al (2012) In vivo imaging of endogenous pancreatic beta-cell mass in healthy and type 1 diabetic subjects using 18F-fluoropropyl-dihydrotetrabenazine and PET. J Nucl Med 53:908-916

17. Bohnen NI, Albin RL, Koeppe RA et al (2006) Positron emission tomography of monoaminergic vesicular binding in aging and Parkinson disease. J Cereb Blood Flow Metab 26:1198-1212

18. Frey KA, Koeppe RA, Kilbourn MR et al (1996) Presynaptic monoaminergic vesicles in Parkinson's disease and normal aging. Ann Neurol 40:873-884

19. Boileau I, Rusjan P, Houle S et al (2008) Increased vesicular monoamine transporter binding during early abstinence in human methamphetamine users: is VMAT2 a stable dopamine neuron biomarker? J Neurosci 28:9850-9856

20. Fagerholm V, Mikkola KK, Ishizu T et al (2010) Assessment of islet specificity of dihydrotetrabenazine radiotracer binding in rat pancreas and human pancreas. J Nucl Med 51:1439-1446 
21. Souza F, Freeby M, Hultman K et al (2006) Current progress in non-invasive imaging of beta cell mass of the endocrine pancreas. Curr Med Chem 13:2761-2773

22. Ichise M, Harris PE (2010) Imaging of beta-cell mass and function. J Nucl Med 51:1001-1004

23. Lin KJ, Weng YH, Wey SP et al (2010) Whole-body biodistribution and radiation dosimetry of 18F-FP-(+)-DTBZ (18F-AV-133): a novel vesicular monoamine transporter 2 imaging agent. J Nucl Med 51:1480-1485

24. Eriksson O, Jahan M, Johnström $P$ et al (2010) In vivo and in vitro characterization of $[18 \mathrm{~F}]-\mathrm{FE}-(+)-\mathrm{DTBZ}$ as a tracer for beta-cell mass. Nucl Med Biol 37:357-363

25. Bormans GM (2010) 11C-Dihydrotetrabenazine beta-cell imaging. J Nucl Med 51:1335-1336

26. Harris PE, Ferrara C, Barba P, Polito T, Freeby M, Maffei A (2008) VMAT2 gene expression and function as it applies to imaging beta-cell mass. J Mol Med (Berl) 86:5-16

27. Kilbourn MR (2010) Rat pancreas uptake of [11C]dihydrotetrabenazine stereoisomers. Nucl Med Biol 37:869-871

28. Virostko J, Henske J, Vinet L et al (2011) Multimodal image coregistration and inducible selective cell ablation to evaluate imaging ligands. Proc Natl Acad Sci U S A 108:2071920724

29. Cline GW, Zhao X, Jakowski AB, Soeller WC, Treadway JL (2011) Islet-selectivity of G-protein coupled receptor ligands evaluated for PET imaging of pancreatic beta-cell mass. Biochem Biophys Res Commun 412:413-418

30. Marselli L, Thorne J, Ahn YB et al (2008) Gene expression of purified beta-cell tissue obtained from human pancreas with laser capture microdissection. J Clin Endocrinol Metab 93:1046-1053

31. Bender FL, Mederos YSM, Li Y et al (2005) The temperaturesensitive ion channel TRPV2 is endogenously expressed and functional in the primary sensory cell line F-11. Cell Physiol Biochem 15:183-194

32. Erickson JD, Eiden LE, Hoffman B (1992) Expression cloning of a reserpine-sensitive vesicular monoamine transporter. Proc Natl Acad Sci USA 89:10993-10997

33. Schafer MK-H, Day R (1995) In situ hybridization histochemistry. Meth Neurosci 23:16-44

34. Weihe E, Schutz B, Hartschuh W, Anlauf M, Schafer MK, Eiden LE (2005) Co-expression of cholinergic and noradrenergic phenotypes in human and non-human autonomic nervous system. J Comp Neurol 492:370-379

35. Kilbourn MR, Hockley B, Lee L et al (2007) Pharmacokinetics of [(18)F]fluoroalkyl derivatives of dihydrotetrabenazine in rat and monkey brain. Nucl Med Biol 34:233-237

36. Ichise M, Vines DC, Gura T et al (2006) Effects of early life stress on [11C]DASB positron emission tomography imaging of serotonin transporters in adolescent peer- and mother-reared rhesus monkeys. J Neurosci 26:4638-4643 